\title{
Bound energy for the exponential-cosine-screened Coulomb potential
}

\author{
Sameer M. Ikhdair* and Ramazan Sever ${ }^{\dagger}$ \\ *Department of Physics, Near East University, Nicosia, North Cyprus, Mersin-10, Turkey \\ ${ }^{\dagger}$ Department of Physics, Middle East Technical University, 06531 Ankara, Turkey.
}

(March 22, 2018)

\begin{abstract}
An alternative approximation scheme has been used in solving the Schrödinger equation for the exponential-cosine-screened Coulomb potential. The bound state energies for various eigenstates and the corresponding wave functions are obtained analytically up to the second perturbation term.

Keywords: Exponential-cosine-screened Coulomb potential, Perturbation theory
\end{abstract}

PACS NO: 03.65.Ge

\section{INTRODUCTION}

The generalized exponential-cosine-screened Coulomb (GECSC) potential or the generalized cosine Yukawa (GCY) potential:

$$
V(r)=-\left(\frac{A}{r}\right) \exp (-\delta r) \cos (g \delta r)
$$

where $A$ is the strength coupling constant and $\delta$ is the screening parameter, is known to describe adequately the effective interaction in many-body enviroment of a variety of fields

\footnotetext{
*sameer@neu.edu.tr

†sever@metu.edu.tr
} 
such as atomic, nuclear, solid-state, plasma physics and quantum field theory [1,2]. It is also used in describing the potential between an ionized impurity and an electron in a metal $[3,4]$ or a semiconductor [5] and the electron-positron interaction in a positronium atom in a solid [6]. The potential in (1) with $g=1$ is known as a cosine-screened Coulomb potential. The static screened Coulomb (SSC) potential ( $g=0$ case) is well represented by Yukawa form: $V(r)=-\left(\alpha Z e^{2}\right) \exp (-\delta r) / r$ which emerges as a special case of the ECSC potential in (1) with $A=\alpha Z e^{2}$, where $\alpha=(137.037)^{-1}$ is the fine-structure constant and $Z$ is the atomic number, is often used for the description of the energy levels of light to heavy neutral atoms [7]. It is known that SSC potential yields reasonable results only for the innermost states when $Z$ is large. However, for the outermost and middle atomic states, it gives rather poor results. Although the bound state energies for the SSC potential with $Z=1$ have been studied [7].

The Schrödinger equation for such a potential does not admit exact solutions. So various approximate methods [8] both numerical and analytical have been developed Hence, the bound-state energies of the ECSC potential were first calculated for the $1 s$ state using numerical $[3,8,9]$ and analytical $[10,11]$ methods and for the $s$ states by a variational method [12]. Additionally, the energy eigenvalues of the ECSC potential [13] have been recalculated for the $1 s$ state with the use of the ground-state logarithmic perturbation theory $[14,15]$ and the Padé approximant method. The problem of determining the critical screening parameter $\delta_{c}$ for the $s$ states was also studied [16].

It has also been shown that the problem of screened Coulomb potentials can be solved to a very high accuracy [17] by using the hypervirial relations $[18,19,20]$ and the Padé approximant method. The bound-state energies of the ECSC potential for all eigenstates were accurately determined within the framework of the hypervirial Padé scheme [21]. Further, the large-N expansion method of Mlodinow and Shatz [22] was also applied to obtain the energies of the ground and first excited $s$ - states and the corresponding wave functions. Recently, we studied the bound-states of the ECSC potential for all states using the shifted large $N$ - expansion technique [23]. 
In this paper, we investigate the bound-state properties of ECSC potential using a new perturbative formalism [24] which has been claimed to be very powerful for solving the Schrödinger equation to obtain the bound-state energies as well as the wave functions in Yukawa or SSC potential problem $[24,25]$ in both bound and continuum regions. This novel treatment is based on the decomposition of the radial Schrödinger equation into two pieces having an exactly solvable part with an addiıtional piece leading to either a closed analytical solution or approximate treatment depending on the nature of the perturbed potential.

The contents of this paper is as follows. In section II we breifly outline the method with all necessary formulae to perform the current calculations. In section III we apply the approach to the Schrödinger equation with the ECSC potential and present the results obtained analytically and numerically for the bound-state energy values. Finally, in section IV we give our concluding remarks.

\section{THE METHOD}

For the consideration of spherically symmetric potentials, the corresponding Schrödinger equation, in the bound state domain, for the radial wave function reads

$$
\frac{\hbar^{2}}{2 m} \frac{\psi_{n}^{\prime \prime}(r)}{\psi_{n}(r)}=V(r)-E_{n}
$$

with

$$
V(r)=\left[V_{0}(r)+\frac{\hbar^{2}}{2 m} \frac{\ell(\ell+1)}{r^{2}}\right]+\Delta V(r),
$$

where $\Delta V(r)$ is a perturbing potential and $\psi_{n}(r)=\chi_{n}(r) u_{n}(r)$ is the full radial wave function, in which $\chi_{n}(r)$ is the known normalized eigenfunction of the unperturbed Schrödinger equation whereas $u_{n}(r)$ is a moderating function corresponding to the perturbing potential. Following the prescription of Ref. 24, we may rewrite (2) as

$$
\frac{\hbar^{2}}{2 m}\left(\frac{\chi_{n}^{\prime \prime}(r)}{\chi_{n}(r)}+\frac{u_{n}^{\prime \prime}(r)}{u_{n}(r)}+2 \frac{\chi_{n}^{\prime}(r) u_{n}^{\prime}(r)}{\chi_{n}(r) u_{n}(r)}\right)=V(r)-E_{n} .
$$


The logarithmic derivatives of the unperturbed $\chi_{n}(r)$ and perturbed $u_{n}(r)$ wave functions are given by

$$
W_{n}(r)=-\frac{\hbar}{\sqrt{2 m}} \frac{\chi_{n}^{\prime}(r)}{\chi_{n}(r)} \quad \text { and } \quad \Delta W_{n}=-\frac{\hbar}{\sqrt{2 m}} \frac{u_{n}^{\prime}(r)}{u_{n}(r)}
$$

which leads to

$$
\frac{\hbar^{2}}{2 m} \frac{\chi_{n}^{\prime \prime}(r)}{\chi_{n}(r)}=W_{n}^{2}(r)-\frac{\hbar}{\sqrt{2 m}} W_{n}^{\prime}(r)=\left[V_{0}(r)+\frac{\hbar^{2}}{2 m} \frac{\ell(\ell+1)}{r^{2}}\right]-\varepsilon_{n},
$$

where $\varepsilon_{n}$ is the eigenvalue for the exactly solvable potential of interest, and

$\frac{\hbar^{2}}{2 m}\left(\frac{u_{n}^{\prime \prime}(r)}{u_{n}(r)}+2 \frac{\chi_{n}^{\prime}(r) u_{n}^{\prime}(r)}{\chi_{n}(r) u_{n}(r)}\right)=\Delta W_{n}^{2}(r)-\frac{\hbar}{\sqrt{2 m}} \Delta W_{n}^{\prime}(r)+2 W_{n}(r) \Delta W_{n}(r)=\Delta V(r)-\Delta \varepsilon_{n}$,

in which $\Delta \varepsilon_{n}=E_{n}^{(1)}+E_{n}^{(2)}+\cdots$ is the correction term to the energy due to $\Delta V(r)$ and $E_{n}=\varepsilon_{n}+\Delta \varepsilon_{n}$. If Eq. (7), which is the most significant piece of the present formalism, can be solved analytically as in (6), then the whole problem, in Eq. (2) reduces to the following form

$$
\left[W_{n}(r)+\Delta W_{n}(r)\right]^{2}-\frac{\hbar}{\sqrt{2 m}}\left(W_{n}(r)+\Delta W_{n}(r)\right)^{\prime}=V(r)-E_{n}
$$

which is a well known treatment within the frame of supersymmetric quantum theory (SSQT) [26]. Thus, if the whole spectrum and corresponding eigenfunctions of the unperturbed interaction potential are known, then one can easily calculate the required superpotential $W_{n}(r)$ for any state of interest leading to direct computation of related corrections to the unperturbed energy and wave function.

For the perturbation technique, we can split the given potential in Eq.(2) into two parts. The main part corresponds to a shape invariant potential, Eq. (6), for which the superpotential is known analytically and the remaining part is treated as a perturbation, Eq. (7). Therefore, it is obvious that ECSC potential can be treated using this prescription. In this case, the zeroth-order term corresponds to the Coulomb potential while higher-order terms consitute the perturbation. However, the perturbation term in its present form cannot 
be solved exactly through Eq. (7). Thus, one should expand the functions related to the perturbation in terms of the perturbation parameter $\lambda$,

$$
\Delta V(r ; \lambda)=\sum_{i=1}^{\infty} \lambda_{i} V_{i}(r), \quad \Delta W_{n}(r ; \lambda)=\sum_{i=1}^{\infty} \lambda_{i} W_{n}^{(i)}(r), \quad E_{n}^{(i)}(\lambda)=\sum_{i=1}^{\infty} \lambda_{i} E_{n}^{(i)},
$$

where $i$ denotes the perturbation order. Substitution of the above expansions into Eq. (7) and equating terms with the same power of $\lambda$ on both sides up to $O\left(\lambda^{3}\right)$ gives

$$
\begin{gathered}
2 W_{n}(r) W_{n}^{(1)}(r)-\frac{\hbar}{\sqrt{2 m}} \frac{d W_{n}^{(1)}(r)}{d r}=V_{1}(r)-E_{n}^{(1)}, \\
W_{n}^{(1) 2}(r)+2 W_{n}(r) W_{n}^{(2)}(r)-\frac{\hbar}{\sqrt{2 m}} \frac{d W_{n}^{(2)}(r)}{d r}=V_{2}(r)-E_{n}^{(2)}, \\
2\left[W_{n}(r) W_{n}^{(3)}(r)+W_{n}^{(1)}(r) W_{n}^{(2)}(r)\right]-\frac{\hbar}{\sqrt{2 m}} \frac{d W_{n}^{(3)}(r)}{d r}=V_{3}(r)-E_{n}^{(3)} .
\end{gathered}
$$

Hence, unlike the other perturbation theories, Eq. (7) and its expansion, Eqs. (10-12), give a flexibility for the easy calculations of the perturbative corrections to energy and wave functions for the nth state of interest through an appropriately chosen perturbed superpotential.

\section{APPLICATION TO THE ECSC POTENTIAL}

Considering the recent interest in various power-law potentials in the literature, we work through the article within the frame of low screening parameter. In this case, the ECSC potential can be expanded in power series of the screening parameter $\delta$ as [10]

$$
V(r)=-\left(\frac{A}{r}\right) \exp (-\delta r) \cos (\delta r)=-\left(\frac{A}{r}\right) \sum_{i=0}^{\infty} V_{i}(\delta r)^{i},
$$

where the perturbation coefficients $V_{i}$ are given by

$$
V_{1}=-1, V_{2}=0, V_{3}=1 / 3, V_{4}=-1 / 6, V_{5}=1 / 30, \cdots .
$$

We now apply this approximation method to the ECSC potential with the angular momentum barrier 


$$
V(r)=-\left(\frac{A}{r}\right) e^{-\delta r} \cos (\delta r)+\frac{\ell(\ell+1) \hbar^{2}}{2 m r^{2}}=\left[V_{0}(r)+\frac{\ell(\ell+1) \hbar^{2}}{2 m r^{2}}\right]+\Delta V(r),
$$

where the first piece is the shape invariant zeroth-order which is an exactly solvable piece corresponding to the unperturbed Coulomb potential with $V_{0}(r)=-A / r$ while $\Delta V(r)=$ $A \delta-\left(A \delta^{3} / 3\right) r^{2}+\left(A \delta^{4} / 6\right) r^{3}-\left(A \delta^{5} / 30\right) r^{4}+\cdots$ is the perturbation term. The literature is rich with examples of particular solutions for such power-law potentials employed in different fields of physics, for recent applications see Refs. $[27,28]$. At this stage one may wonder why the series expansion is truncated at a lower order. This can be understood as follows. It is widely appreciated that convergence is not an important or even desirable property for series approximations in physical problems. Specifically, a slowly convergent approximation which requires many terms to achieve reasonable accuracy is much less valuable than the divergent series which gives accurate answers in a few terms. This is clearly the case for the ECSC problem [29]. However, it is worthwhile to note that the main contributions come from the first three terms. Thereby, the present calculations are performed up to the second-order involving only these additional potential terms, which suprisingly provide highly accurate results for small screening parameter $\delta$.

\section{A. Ground State Calculations $(n=0)$}

In the light of Eq. (6), the zeroth-order calculations leading to exact solutions can be carried out readily by setting the ground-state superpotential and the unperturbed exact energy as

$$
W_{n=0}(r)=-\frac{\hbar}{\sqrt{2 m}} \frac{\ell+1}{r}+\sqrt{\frac{m}{2}} \frac{A}{(\ell+1) \hbar}, \quad E_{n}^{(0)}=-\frac{m A^{2}}{2 \hbar^{2}(n+\ell+1)^{2}}, \quad n=0,1,2, \ldots
$$

and from the literature, the corresponding normalized Coulomb bound-state wave function $[30]$

$$
\chi_{n}(r)=N_{n, l}^{(C)} r^{\ell+1} \exp [-\beta r] \times L_{n}^{2 \ell+1}[2 \beta r],
$$


in which $N_{n, l}^{(C)}=\left[\frac{2 m A}{(n+\ell+1) \hbar^{2}}\right]^{\ell+1} \frac{1}{(n+\ell+1)} \frac{1}{\sqrt{\frac{\hbar^{2}}{m A n !}(n+2 \ell+1) !}}$ is a normalized constant, $\beta=\frac{m A}{(n+\ell+1) \hbar^{2}}$ and $L_{n}^{k}(x)=\sum_{m=0}^{n}(-1)^{m} \frac{(n+k) !}{(n-m) !(m+k) ! m !} x^{m}$ is an associate Laguarre polynomial function [31].

For the calculation of corrections to the zeroth-order energy and wavefunction, one needs to consider the expressions leading to the first- and second-order perturbation given by Eqs. (10-12). Multiplication of each term in these equations by $\chi_{n}^{2}(r)$, and bearing in mind the superpotentials given in Eq. (5), one can obtain the straightforward expressions for the first-order correction to the energy and its superpotential:

$$
E_{n}^{(1)}=\int_{-\infty}^{\infty} \chi_{n}^{2}(r)\left(-\frac{A \delta^{3}}{3} r^{2}\right) d r, W_{n}^{(1)}(r)=\frac{\sqrt{2 m}}{\hbar} \frac{1}{X_{n}^{2}(r)} \int^{r} \chi_{n}^{2}(x)\left[E_{n}^{(1)}+\frac{A \delta^{3}}{3} x^{2}\right] d x,
$$

and also for the second-order correction and its superpotential:

$$
\begin{gathered}
E_{n}^{(2)}=\int_{-\infty}^{\infty} \chi_{n}^{2}(r)\left[\frac{A \delta^{4}}{6} r^{3}-W_{n}^{(1) 2}(r)\right] d r \\
W_{n}^{(2)}(r)=\frac{\sqrt{2 m}}{\hbar} \frac{1}{X_{n}^{2}(r)} \int^{r} \chi_{n}^{2}(x)\left[E_{n}^{(2)}+W_{n}^{(1) 2}(x)-\frac{A \delta^{4}}{6} x^{3}\right] d x,
\end{gathered}
$$

for any state of interest. The above expressions calculate $W_{n}^{(1)}(r)$ and $W_{n}^{(2)}(r)$ explicitly from the energy corrections $E_{n}^{(1)}$ and $E_{n}^{(2)}$ respectively, which are in turn used to calculate the moderating wave function $u_{n}(r)$.

Thus, through the use of Eqs. (18) and (19), after some lengthy and tedious integrals, we find the zeeroth order energy shift and their moderating superpotentials as

$$
\begin{gathered}
E_{0}^{(1)}=-\frac{\hbar^{4}(\ell+1)^{2}(\ell+2)(2 \ell+3)}{6 A m^{2}} \delta^{3}, \\
E_{0}^{(2)}=\frac{\hbar^{6}(\ell+1)^{3}(\ell+2)(2 \ell+3)(2 \ell+5)}{24 A^{2} m^{3}} \delta^{4} \\
-\frac{\hbar^{10}(\ell+1)^{6}(\ell+2)(2 \ell+3)\left(8 \ell^{2}+37 \ell+43\right)}{72 A^{4} m^{5}} \delta^{6}, \\
W_{0}^{(1)}(r)=-\frac{\hbar(\ell+1) \delta^{3} r}{3 \sqrt{2 m}}\left\{r+\frac{\hbar^{2}(\ell+1)(\ell+2)}{A m}\right\}, \\
W_{0}^{(2)}(r)=-\frac{\hbar \delta^{4} c r}{2 \sqrt{2 m}}\left\{\delta^{2} r^{3}+a r^{2}+b\left[r+\frac{\hbar^{2}(\ell+1)(\ell+2)}{A m}\right]\right\}-\frac{\hbar(\ell+1)}{\sqrt{2 m} A} E_{0}^{(2)},
\end{gathered}
$$


in which

$$
\begin{aligned}
& a=\frac{\hbar^{2}(\ell+1)(3 \ell+7) \delta^{2}}{A m}-\frac{3 A m}{\hbar^{2}(\ell+1)^{2}}, \quad b=\left[\frac{\hbar^{4}(\ell+1)^{2}\left(8 \ell^{2}+37 \ell+43\right) \delta^{2}}{2 A^{2} m^{2}}-\frac{3}{2} \frac{(2 \ell+5)}{(\ell+1)}\right] \\
& \mathrm{c}=\frac{\hbar^{2}(\ell+1)^{3}}{9 A m}
\end{aligned}
$$

Therefore, the analytical expressions for the lowest energy and full radial wave function of an ECSC potential are then given by

$$
E_{n=0, \ell}=E_{n=0, \ell}^{(0)}+A \delta+E_{0}^{(1)}+E_{0}^{(2)}+\cdots, \psi_{n=0, \ell}(r) \approx \chi_{n=0, \ell}(r) u_{n=0, \ell}(r),
$$

in which

$$
u_{n=0, \ell}(r) \approx \exp \left(-\frac{\sqrt{2 m}}{\hbar} \int^{r}\left(W_{0}^{(1)}(x)+W_{0}^{(2)}(x)\right) d x\right)
$$

Hence, the explicit form of the full wave function in (22) for the ground state is

$$
\psi_{n=0, \ell}(r)=\left[\frac{2 m A}{(\ell+1) \hbar^{2}}\right]^{\ell+1} \frac{1}{(\ell+1)^{2}} \sqrt{\frac{A m}{\hbar^{2}(2 \ell+1) !}} r^{\ell+1} \exp (P(r)),
$$

with $P(r)=\sum_{i=1}^{5} p_{i} r^{i}$ is a polynomial of fifth order having the following coefficients:

$p_{1}=\frac{(\ell+1)}{A} E_{0}^{(2)}-\frac{A m}{(\ell+1) \hbar^{2}}, \quad p_{2}=\frac{9}{4} \frac{(\ell+2)}{(\ell+1)^{2}} c^{2} d \delta^{4}, \quad p_{3}=\frac{1}{6} c d \delta^{4}, \quad p_{4}=\frac{1}{8} a c \delta^{4}, p_{5}=\frac{1}{10} c \delta^{6}$,

in which $d=b+\frac{6 A m}{\hbar^{2}(\ell+1)^{2} \delta}$ and other parameters are given in $(21)$.

\section{B. Excited state calculations $(n \geq 1)$}

The calculations procedures lead to a handy recursion relations in the case of ground states, but becomes extremely cumbersome in the description of radial excitations when nodes of wavefunctions are taken into account, in particular during the higher order calculations. Although several attempts have been made to bypass this difficulty and improve

calculations in dealing with excited states, (cf. e.g. [32], and the references therein) within the frame of supersymmetric quantum mechanics. 
Using Eqs. (5) and (17), the superpotential $W_{n}(r)$ which is related to the excited states can be readily calculated through Eqs. (18) and (19). So the first-order corrections in the first excited state $(n=1)$ are

$$
\begin{gathered}
E_{1}^{(1)}=-\frac{\hbar^{4}(\ell+2)^{2}(\ell+7)(2 \ell+3)}{6 A m^{2}} \delta^{3}, \\
W_{1}^{(1)}(r) \approx-\frac{\hbar(\ell+2) \delta^{3} r}{3 \sqrt{2 m}}\left\{r+\frac{\hbar^{2}(\ell+2)(\ell+3)}{A m}\right\} .
\end{gathered}
$$

Consequently, the use of $W_{1}^{(1)}(r)$ in the preceeding equation in (19) gives the energy correction in the second-order as

$$
\begin{aligned}
E_{1}^{(2)} \approx & \frac{\hbar^{6}(\ell+2)^{3}(\ell+11)(2 \ell+3)(2 \ell+5)}{24 A^{2} m^{3}} \delta^{4} \\
& -\frac{\hbar^{10}(\ell+2)^{6}(\ell+3)(2 \ell+3)\left(7 \ell^{2}+101 \ell+211\right)}{72 A^{4} m^{5}} \delta^{6}
\end{aligned}
$$

Therefore, the approximated energy value of the ECSC potential corresponding to the first excited state is

$$
E_{n=1, \ell}=E_{1}^{(0)}+A \delta+E_{1}^{(1)}+E_{1}^{(2)}+\cdots
$$

The related radial wavefunction can be expressed in an analytical form in the light of Eqs (18), (19) and (22), if required. The appromation used in this work would not affect considerably the sensitivity of the calculations. On the other hand, it is found analytically that our investigations put forward an interesting hierarchy between $W_{n}^{(1)}(r)$ terms of different quantum states in the first order after circumventing the nodal difficulties elegantly,

$$
W_{n}^{(1)}(r)=-\frac{\hbar(n+\ell+1) \delta^{3} r}{3 \sqrt{2 m}}\left\{r+\frac{\hbar^{2}(n+\ell+1)(n+\ell+2)}{A m}\right\},
$$

which, for instance, for the second excited state $(n=2)$ leads to the first-order correction

$$
\begin{gathered}
E_{2}^{(1)}=-\frac{\hbar^{4}(\ell+3)^{2}(\ell+2)(2 \ell+23)}{6 A m^{2}} \delta^{3}, \\
W_{2}^{(1)}(r)=-\frac{\hbar(\ell+3) \delta^{3} r}{3 \sqrt{2 m}}\left\{r+\frac{\hbar^{2}(\ell+3)(\ell+4)}{A m}\right\} .
\end{gathered}
$$


Thus, the use of $W_{2}^{(1)}(r)$ in the preceeding equation (19) gives the energy correction in the second-order as

$$
\begin{aligned}
E_{2}^{(2)}= & \frac{\hbar^{6}(\ell+2)(\ell+3)^{2}(2 \ell+5)\left(2 \ell^{2}+45 \ell+153\right)}{24 A^{2} m^{3}} \delta^{4} \\
& -\frac{\hbar^{10}(\ell+2)(\ell+3)^{5}\left(16 \ell^{4}+474 \ell^{3}+3879 \ell^{2}+12118 \ell+12873\right)}{72 A^{4} m^{5}} \delta^{6} .
\end{aligned}
$$

Therefore, the approximated energy eigenvalue of the ECSC potential corresponding to the second excited state is

$$
E_{n=2, \ell}=E_{2}^{(0)}+A \delta+E_{2}^{(1)}+E_{2}^{(2)}+\cdots
$$

For the numerical work, some numerical values of the perturbed energies of the $1 s$ and $2 s$ states, in the atomic units we take $\hbar=m=A=1$, for different values of the screening parameter $\delta$ in the range $0 \leq \delta \leq 0.10$ are displayed in Tables 1 and 2 , respectively. The results are consistent to order $\delta^{6}$ with earlier results obtained by applying different methods in Refs. [9,22,23]. Further, we display the results for the energy eigenvalues of $2 s, 2 p, 3 s$, $3 p$, and $3 d$ states in Tables 3 and 4 . Our results are then compared with accurate energy eigenvalues obtained by other authors. Thus, through the comparison of our results with those of Refs. [9,10,22,23] for large $n$ and $\ell$ - values and small screening parameter values yields indeed excellent results.

On the other hand, we take $A=\sqrt{2}$ and $\delta=\sqrt{2} G$. Cosequently, we compute the binding energies $\left(-E_{n, \ell}\right)$ of the lowest-lying states, $1 s$ to $3 d$, for various values of $\delta$. Hence, the detailed analysis of the results in terms of various domains of parameters $A$ and $\delta$ of ECSC potential are displayed in Table 5. For further study of the bound-state energies and normalizations with analytical perturbation calculation in Table 6 . We consider $A=Z=4$, $8,16,24$ in order to cover the range of low to high atomic numbers. For low strength of $A=Z$, the energy eigenvalues nobtained are in good agreement with the other methods for low values of the screening parameter $\delta$. Obviously, when $\delta$ is small the Coulomb field character prevails and the method has been adjusted to that. However, the results become gradually worse as $A$ and/or $\delta$ are large. 


\section{CONCLUDING REMARKS}

We have shown that the bound-state energies of the exponential cosine screened Coulomb (ECSC) potential for all eigenstates can be accurately determined within the framework of a new approximation formalism. Avoiding the disadvantages of the standard non-relativistic perturbation theories, the present formulae have the same simple form both for ground and excited states and provide, in principle, the calculation of the perturbation corrections up to any arbitrary order in analytical or numerical form.

Additionally, the application of the present technique to ECSC potential is really of great interest leading to analytical expressions for both energy eigenvalues and wave functions. Comparing various energy levels with different works in the literature we find that this

treatment is quite reliable and further analytical calculations with this non-perturbative scheme would be useful. In particular, the method becomes more reliable as the potential strength increases.

\section{ACKNOWLEDGMENTS}

S.M. Ikhdair wishes to dedicate this work to his son Musbah for his love and assistance. 


\section{REFERENCES}

[1] P. Anderson, Phys. Rev. 86, 694 (1952); R. Kubo, Phys. Rev. 87, 568 (1952).

[2] R. A. Ferrell and D. J. Scalapino, Phys. Rev. A 9, 846 (1974); A. J. Bray, J. Phys. A 7 , 2144 (1974); E. Brezin, J. Phys. A 12, 759 (1979).

[3] V. L. Bonch-Bruevich and V. B. Glasko, Sov. Phys. Dokl. 4, 147 (1959).

[4] N. Takimoto, J. Phys. Soc. Jpn. 14, 1142 (1959).

[5] V. L. Bonch-Bruevich and Sh. M. Kogan, Sov. Phys. Solid State 1,1118 (1960) C. Weisbuch and B. Vinter, Quantum Semiconductor Heterostructures (Academic Press, New York, 1993); P. Harrison, Quantum Wells, Wires and Dots (John Wiley and Sons, England, 2000).

[6] E. P. Prokopev, Sov. Phys. Solid State 9, 993 (1967).

[7] R. Dutt and Y. P. Varshni, Z. Phys. D 2, 207 (1986).

[8] R. Dutt, A. Ray and P. P. Ray, Phys. Lett. A 83, 65 (1981); C. S. Lam and Y. P. Varshni, Phys. Rev. A 4, 1875 (1971); D. Singh and Y. P. Varshni, Phys. Rev. A 28, 2606 (1983).

[9] H. de Meyer et al., J. Phys. A 18, L 849 (1985).

[10] C. S. Lai, Phys. Rev. A 26, 2245 (1982).

[11] R. Dutt et al., J. Phys. A 18, 1379 (1985).

[12] C. S. Lam and Y. P. Varshni, Phys. Rev. A 6, 1391 (1972).

[13] V. L. Eletsky, V. S. Popov, and V. M. Weinberg, Phys. Lett. A 84, 235 (1981).

[14] A. D. Dolgov and V. S. Popov, Phys. Lett. B 79, 403 (1978).

[15] Y. Aharanov and C. K. Au, Phys. Rev. Lett. 42, 1582 (1979) 
[16] P. P. Ray and A. Ray, Phys. Lett. B 78, 443 (1981).

[17] C. S. Lai, Phys. Rev. A 23, 455 (1981).

[18] J. D. Hirschfelder, J. Chem. Phys. 33, 1462 (1960).

[19] J. Killingbeck, Phys. Lett. A 65, 87 (1978).

[20] M. Grant and C. S. Lai, Phys. Rev. A 20, 718 (1979).

[21] C. S. Lai, Phys. Rev. A 26, 2245 (1982).

[22] R. Sever and C. Tezcan, Phys. Rev. A 35, 2725 (1987).

[23] S. M. Ikhdair and R. Sever, Z. Phys. D 28,1 (1993).

[24] B. Gönül, K. Köksal and E. Bakır, [arXiv:quant-ph/0507098]; B. Gönül, Chin. Phys. Lett. 21, 1685 (2004).

[25] B. Gönül, Chin. Phys. Lett. 21, 2330 (2004); B. Gönül and M. Koçak, Mod. Phys. Lett. A 20, 355 (2005); B. Gönül, N. Çelik and E. Olğar, Mod. Phys. Lett. A 20, 1683 (2005); B. Gönül and M. Koçak, Chin. Phys. Lett. 20, 2742 (2005); ibid. Mod. Phys. Lett. A 20, $1983(2005)$.

[26] F. Cooper, A. Khare and U. P. Sukhatme, Phys. Rep. 251, 267 (1995).

[27] M. Zonjil, J. Math. Chem. 26, 157 (1999).

[28] M. Alberg and L. Wilets, Phys. Lett. A 286, 7 (2001).

[29] D. J. Doren and D. R. Herschbach, Phys. Rev. A 34, 2665 (1986).

[30] H. A. Bethe and E. E. Salpeter, Quantum Mechanics of One- and Two-Electron Atoms (springer, Berlin, 1957).

[31] L. S. Gradshteyn and I. M. Ryzhik, Tables of Integrals, Series and Products (Academic, New York, 1965). 
[32] C. Lee, Phys. Lett. A 267, 101 (2000). 


\section{TABLES}

TABLE I. Comparison of bound energy eigennvalues for $0 \leq \delta \leq 0.1$ for the $1 s$ state in atomic units.

\begin{tabular}{|c|c|c|c|c|}
\hline$\delta$ & $1 / N[22]$ & Dynamical [9] & Shifted $1 / N[23]$ & $E_{n, \ell}$ \\
\hline 0.01 & -0.490001 & -0.4900010 & & -0.4900009 \\
\hline 0.02 & -0.480008 & -0.4800078 & -0.48000783 & -0.4800078 \\
\hline 0.03 & -0.470026 & -0.4700260 & & -0.4700259 \\
\hline 0.04 & -0.460061 & -0.4600609 & -0.46006101 & -0.4600608 \\
\hline 0.05 & -0.450117 & -0.4501174 & & -0.4501172 \\
\hline 0.06 & -0.440200 & -0.4402004 & -0.44020057 & -0.4402000 \\
\hline 0.07 & -0.430313 & & & -0.4303134 \\
\hline 0.08 & -0.420461 & -0.4204636 & -0.42046386 & -0.4204617 \\
\hline 0.09 & -0.410647 & & & -0.4106488 \\
\hline 0.1 & -0.400875 & -0.4008839 & -0.40088421 & -0.4008785 \\
\hline
\end{tabular}


TABLE II. Comparison of bound energy eigennvalues for $0 \leq \delta \leq 0.1$ for the $2 s$ state in atomic units.

\begin{tabular}{|c|c|c|c|c|}
\hline$\delta$ & $1 / N[22]$ & Dynamical [9] & Shifted $1 / N[23]$ & $E_{n, \ell}$ \\
\hline 0.01 & -0.115013 & -0.1150135 & & -0.1150134 \\
\hline 0.02 & -0.105103 & -0.1051036 & -0.10510361 & -0.1051033 \\
\hline 0.03 & -0.095334 & -0.0953366 & & -0.0953346 \\
\hline 0.04 & -0.085755 & -0.0857690 & -0.08576959 & -0.0857621 \\
\hline 0.05 & -0.076406 & -0.0764497 & & -0.0764326 \\
\hline 0.06 & -0.067311 & -0.0674217 & -0.06742608 & -0.0673900 \\
\hline 0.07 & -0.058482 & & & -0.0586800 \\
\hline 0.08 & -0.049915 & -0.0503922 & -0.05040825 & -0.0503576 \\
\hline 0.09 & -0.041598 & & & -0.0424945 \\
\hline 0.1 & -0.033500 & -0.0349677 & -0.03500467 & -0.0351880 \\
\hline
\end{tabular}


TABLE III. Energy eigenvalues as a function of screening parameter $\delta$ for the $2 s$ and $2 p$ states in atomic units.

\begin{tabular}{lrrrlllll}
\hline \hline State $\delta$ & $E[10,10][10]$ & $E[10,11][10]$ & Pertur. $[10]$ & Variational $[10]$ & Shifted $[23]$ & $E_{n, \ell}$ \\
\hline $2 s$ & 0.10 & -0.034941 & -0.034941 & -0.034425 & -0.034935 & -0.03500467 & -0.0351880 \\
$2 p$ & & -0.032469 & -0.032469 & -0.032042 & -0.03247015 & -0.0326733 \\
$2 s$ & 0.08 & -0.050387 & -0.050387 & -0.050222 & -0.050384 & -0.05040825 & -0.0503576 \\
$2 p$ & & -0.048997 & -0.048997 & & & -0.04899693 & -0.0489939 \\
$2 s$ & 0.06 & -0.067421 & -0.067421 & $-0.067385-0.067421$ & -0.06742608 & -0.0673900 \\
$2 p$ & -0.066778 & -0.066778 & & & -0.06677729 & -0.0667611 \\
$2 s$ & 0.04 & -0.085769 & -0.085769 & -0.085767 & -0.085769 & -0.08576959 & -0.0857621 \\
$2 p$ & -0.085591 & -0.085591 & & -0.08555913 & -0.0855520 \\
$2 s$ & 0.02 & -0.105104 & -0.105104 & $-0.105104-0.105104$ & -0.10510361 & -0.1051033 \\
$2 p$ & -0.105075 & -0.105075 & -0.105075 & -0.10507464 & -0.1050744 \\
\hline \hline
\end{tabular}


TABLE IV. Energy eigenvalues as a function of screening parameter $\delta$ for the $3 s, 3 p$ and $3 d$ states in atomic units.

\begin{tabular}{|c|c|c|c|c|c|c|c|}
\hline State & $\delta$ & $E[10,10][10]$ & $E[10,11][10]$ & Pertur. [10] & Variational [10] & Shifted [23] & $E_{n, \ell}$ \\
\hline $3 s$ & 0.06 & -0.005461 & -0.005462 & -0.004538 & -0.005454 & -0.00566638 & -0.0070778 \\
\hline $3 p$ & & -0.004471 & -0.004472 & & & -0.00449233 & -0.0054058 \\
\hline $3 d$ & & -0.002308 & -0.002309 & & & -0.00231356 & -0.0029240 \\
\hline $3 s$ & 0.05 & -0.011576 & -0.011576 & & & -0.01168544 & -0.0119523 \\
\hline $3 p$ & & -0.010929 & -0.010929 & -0.010538 & & -0.01093985 & -0.0111117 \\
\hline $3 d$ & & -0.009555 & -0.009555 & -0.009292 & & -0.00955542 & -0.0096940 \\
\hline $3 s$ & 0.04 & -0.018823 & -0.018823 & -0.018707 & -0.018822 & -0.01886716 & -0.0188586 \\
\hline $3 p$ & & -0.018453 & -0.018453 & & & -0.01845705 & -0.0184505 \\
\hline $3 d$ & & -0.017682 & -0.017682 & & & -0.01768208 & -0.0176910 \\
\hline $3 s$ & 0.02 & -0.036025 & -0.036025 & -0.036022 & -0.036025 & -0.03602738 & -0.0360213 \\
\hline $3 p$ & & -0.035968 & -0.035968 & -0.035965 & & -0.03596771 & -0.0359640 \\
\hline $3 d$ & & -0.035851 & -0.035851 & -0.035849 & & -0.03585066 & -0.0358490 \\
\hline
\end{tabular}

TABLE V. Energy eigenvalues of the ECSC potential in units of $\hbar=m=1, A=2^{1 / 2}$ and $\delta=G A$.

\begin{tabular}{lcccccc}
\hline \hline $\mathrm{G}$ & State $-E_{0,0}$ & State $-E_{1,0}$ & State $-E_{0,1}$ & \multicolumn{1}{c}{ State $-E_{1,1}$} & \multicolumn{2}{l}{ State $-E_{0,2}$} \\
\hline $0.0021 s$ & $0.99600002 s$ & $0.24600022 p$ & $0.24600013 p$ & $0.10711203 d$ & 0.1071114 \\
0.005 & 0.9900002 & 0.2400034 & 0.2400024 & 0.1011255 & 0.1011160 \\
0.010 & 0.9800019 & 0.2300269 & 0.2300193 & 0.0912217 & 0.0911475 \\
0.020 & 0.9600156 & 0.2102066 & 0.2101489 & 0.0719281 & 0.0713617 \\
0.025 & 0.9500302 & 0.2003953 & 0.2002857 & 0.0626485 & 0.0615665 \\
0.050 & 0.9002344 & 0.1528652 & 0.1520991 & 0.0222235 & 0.0141374 \\
\hline \hline
\end{tabular}


TABLE VI. Energy eigenvalues of the ECSC potential for all states in units of $\hbar=2 m=1$, and $\delta=0.2 \mathrm{fm}^{-1}$.

\begin{tabular}{|c|c|c|c|c|c|c|c|}
\hline$A$ & $\ell$ & $n$ & $-E_{n, \ell}$ & $A$ & $\ell$ & $n$ & $-E_{n, \ell}$ \\
\hline 4 & 0 & 0 & 3.207029 & 16 & 0 & 1 & 12.825303 \\
\hline \multirow[t]{2}{*}{8} & 0 & 0 & 14.403752 & & 0 & 2 & 4.023139 \\
\hline & 1 & 0 & 2.433587 & & 1 & 1 & 4.009505 \\
\hline \multirow[t]{2}{*}{16} & 0 & 0 & 60.801938 & 24 & 0 & 1 & 31.217455 \\
\hline & 1 & 0 & 12.818287 & & 0 & 2 & 11.279786 \\
\hline \multirow[t]{4}{*}{24} & 0 & 0 & 139.20131 & & 1 & 1 & 11.269899 \\
\hline & 1 & 0 & 31.212563 & & 1 & 2 & 4.412177 \\
\hline & 2 & 0 & 11.249961 & & 2 & 1 & 4.380887 \\
\hline & & & & & 2 & 2 & 1.411568 \\
\hline
\end{tabular}

